\title{
MAPUCHES EN LA MIRA DE LAS RACIONALIDADES POLITICAS.
}

Poder y Diferencia Cultural desde la Conquista Española hasta el Chile Actual.

Mapuches on the overlook of Political Racionalities. Power and Cultural Difference from Spanish Conquiest to Actual Chile Olaf Kaltmeier 1

\section{RESUMEN}

En este artículo exploramos las perspectivas de la utilización de los conceptos de poder y dominación de Michel Foucault aplicándolos a la relaciones inter-étnicas entre los mapuche y los conquistadores españoles y el Estado chileno respectivamente. No sólo fijándonos en la diferencia entre el poder soberano y el poder disciplinario proponemos abrir la discusión con el concepto foucauldiano de la gubernamentalidad hacía múltiples formaciones de poder históricas-espaciales. En este sentido podemos distinguir las formaciones siguientes según sus racionalidades políticas: poder soberano, poder pastoral y razón del Estado, la comunidad pura, la sociedad disciplinada, gubernamentalidad neoliberal.

\section{Palabras clave}

racionalidades políticas, poder, diferencia cultural, mapuches, Conquista española, Chile actual

Trabajo recepcionado : julio 2004

Trabajo aceptado : agosto 2005 


\section{ABSTRACT}

This article explores the perspectives of the use of a foucauldian analisis of power and domination in the case of the inter-ethnic relations between the indigenous mapuche people and first the spanish conquerers and second the chilenean state. It is suggested not to fix on the early foucauldian distinction between souvereign and disciplinary power but to open the analsis to historical and spatial specific formations of power and domination with help of the late foucauldian concept of governmentality. In this sense, focussing on the political rationality, we can distinguish between these formations: souvereign power, pastoral power- state reason, the pure community, disciplinary society, neoliberal governmentality.

\section{Keys words}

Political racionalities, power, cultural difference, Spanish Conquiest, Actual Chile, mapuche on the overlook

\section{INTRODUCCIÓN}

Objeto de este artículo son las complejas y conflictivas relaciones entre los mapuches por un lado y los conquistadores españoles, seguidos por el Estado chileno por otro. Partimos de la hipótesis de que podemos distinguir formaciones histórico-espaciales contingentes que no se caracterizan por sus rasgos económicos, mentales o según los cambios de sistemas políticos sino por sus conceptualizaciones y programas específicos de poder y dominación.

Consideramos que los conceptos que se refieren en los trabajos de Michel Foucault son sumamente fructíferos para tal perspectiva, no reduciéndolos a un esquematismo a-histórico, sino poniéndolos en su contexto. En los trabajos de Guillaume Boccara encontramos muchos antecedentes para la aplicación de los conceptos foucauldianos a la situación colonial que sufrió el pueblo reche-mapuche.

Boccara se refiere sobre todo a la distinción entre poder soberano y disciplinario. Sin embargo, el mismo Foucault se distancia en una crisis intelectual de su antigua oposición de poder soberano y poder disciplinario. introduciendo el concepto de gobernar.2 En este contexto surgió en los trabajos de Foucault el neologismo gouvernementalité/ gubernamentalidad. Este neologismo de Foucault, que consta de las dos componentes "gobernar" y "mentalidad", caracteriza una relación complementaria entre poder y la subjetividad que es típica para los regímenes modernos. Vinculando las macro-estructuras (el Estado) con las micro-estructuras (el sujeto), Foucault soslaya el campo de las esencias (el

2. Foucault criticó el análisis tradicional (incluso marista) por entender poder sólo en términos del poder soberano, es decir bajo la matriz de derecho/sumisión/represión poniendo énfasis en la creatividad y la subterraneidad del poder. En este contexto introdujo el concepto del poder disciplinario con su relacionalidad, sus micro-físicas actuando bajo la lógica de la guerra (Foucault 1999: 45-46, 1992a). Pero el concepto del poder disciplinario es teóricamente opuesto del concepto del poder soberano. moviéndose de esta manera en el mismo margen. En "La voluntad de saber "se distanció Foucault de su conceptualización del poder soberano en términos juridicos recalcando el derecho de deducción cuyo extremo es el derecho de matar. En contra este poder se formó en el siglo XVII y XVIII un bio-poder asestando al cuerpo (disciplina) y al cuerpo de la sociedad (biopolítica). (Foucault 1994b: 16) Esto abrió el camino al descubrimiento del tercer tipo de poder, la gubernamentalidad. 
Estado y el sujeto) y vislumbra, más bien, una tercera dimensión, aquella de las formas de gobernar con sus respectivas formas de estructuración del comportamiento, mostrando que el dominio alcanza su máxima expresión de eficiencia cuando logra estructurar las mentalidades de los sujetos para que éstos se dominen a sí mismos.

Foucault rechaza, asi, una historia de instituciones que las esencializa, proponiendo en vez de dicha historia pensarlas desde afuera. descentrarlas. En el caso de instituciones como el hospital, la escuela, la cárcel, esta perspectiva desde afuera es la disciplina y en el caso del Estado son éstas las técnicas de gobernar. Así, reemplaza la característica interna de la función por la característica externa de las estrategias y tácticas enfocando así las relaciones de poder.

Eso no quiere decir que en vez del dualismo ahora simplemente podamos hablar de la trinidad "poder soberano-disciplina-gobernar" sino que recalcamos la importancia de contextualizar el análisis de poder y tomar en cuenta la diversidad de formaciones históricas de poder y dominación.

Son precisamente los estudios genealógicos de Foucault, que nos muestran que estos tipos de poder no son elementos cuasi-ontológicos sino construcciones sociales en un contexto históricoespacial. Esto no significa que apuntemos a una lógica de desarrollo en cuanto a qué tipos antiguos de poder sean constituidos por nuevos, más "modernos".3 Tampoco se trata de encontrar esquemáticamente la receta de la mezcla de los tres tipos de poder. Más bien se trata de tres movimientos que se influencian y transforman mutuamente. Por eso creemos que existen formas diferentes de poder y dominación que obedecen a racionalidades propias.

Adhiriendo a este análisis de poder contextualizado queremos en lo siguiente distinguir formaciones de dominación históricoespaciales. Según Foucault el poder está en todas las partes de la sociedad, y se habla de dominación cuando el poder es permanente y está institucionalizado con medidas económicas, políticas, militares estableciendo una asimetría estable (Foucault, 1985: 20). Aplicando el concepto de formación de dominación no apuntamos al análisis de estados reales sino a racionalidades políticas, a "programas". La noción de formación hace alusión a la formación discursiva de Foucault y a la formación de Estado de la teoría marxista. En nuestro enfoque al poder, éste se caracteriza por discursos. dispositivos y gubernamentalidades que definen el orden de lo enunciable; es visible el campo de la acción.

En el centro de nuestro análisis están las relaciones inter-étnicas de los mapuches en Chile desde la Conquista hasta el fin del milenio. Michel Foucault desarrolló sus teorías aplicándolas a Europa occidental. El no se enfrentó con situaciones coloniales o poscoloniales, aparte de sus trabajos periodísticos sobre la revolución irania (ver Lemke, 2002: 7399). Surge por esto la pregunta sobre la aplicabilidad de sus conceptos a dichas realidades.4 Partimos de la idea de que a causa de la génesis del sistema capitalista mundial se globalizaron en el "longue durée del siglo XVI" ciertas técnicas de poder junto con la economía.

Históricamente, la relación entre mapuches y conquistadores españoles y, actualmente, la relación del Estado chileno está marcada por conflictos, luchas y procesos dialécticos de constitución propia y ajena. Tenemos que recalcar que procesos de dominación y de poder no son determinantes - lo que será al fin un modelo mecánico, monocausal - sino que existen también procesos de constitución propia individual y colectiva. Si pensamos el poder de una manera relacional. también hay que integrar procesos de resistencia sistemática. Esta resistencia no puede ser deducida de las relaciones de poder, ni puede ser ontologizada. Debido al limitado espacio disponible, no podemos en esta contribución analizar adecuadamente los procesos de resistencia mapuche, por lo que sólo nos permitimos insinuarios.

\footnotetext{
3. Foucault advirtió poner la trinidad de tipos de poder en una línea de cierto desarrollo histórico. En lugar de tratarse de un cambio de diferentes tipos, se trata de un triángulo. (Foucault 2004a: 161). A nuestro modo de ver foucault toma posición con esta advertencia contra conceptos idealistas modernos (Hegel) de progreso y desarrollo.

4. En el caso de los mapuches contamos con los trabajos excelentes de Guillaume Boccara sabre la fase colonial, aunque Boccara se queda dentro del dualismo entre poder soberano y poder disciplinario (1998, 1999).
} 


\section{Metodología analítica}

Poder soberano

En la primera etapa de la Conquista de lo que hoy día llamamos Chile (1541-1650) accionaban los conquistadores españoles bajo la lógica del poder soberano. Trataron de someter a los indígenas al poder del soberano español. En primer lugar, Michel Foucault pensaba el poder soberano como acoplamiento de poder y derecho. Este era caracterizado por la binaridad del código jurídico, diferenciando entre lo correcto y lo falso, lo legítimo y lo ilegítimo. El soberano era el centro simbólico (Foucault, 1999: 34.) siendo también el rey español la figura central de la administración de las colonias (ver Muñoz, 1986: 119).5 La fundación de los vicereinatos y las audiencias, como la Audiencia de Chile, fundada en 1563, representaron al soberano ausente.

En "La voluntad de saber", esta concepción jurídica del poder está sustituida por una conceptualización bio-política en torno al derecho a la deducción. El aspecto central es ahora el dominio del soberano sobre la vida y la muerte. El derecho de quitar la vida es el extremo de un poder que obedece a la lógica de la deducción. Se ejercía como "instancia de deducción, mecanismo de sustracción, derecho de apropiarse de una parte de las riquezas, extorsión de productos, de bienes, de servicios, de trabajo y de sangre, impuesto a los súbditos." (Foucault, 1998b: 164) Elementos de la concepción jurídica en el marco de la concepción bio-política pueden ser encontrados también en la Conquista. Además encontramos en la formación soberana un discurso que no establece un saber sino más bien un no-saber sobre el otro, percibiendo a los mapuche como imagen negativa de lo propio: "sans loi, sans roi, sans foi" (Boccara, 1999a: 85). Esta triple ausencia de un dios omnipotente, de un rey y de una ley - todos elementos claves del poder soberano era hasta mitades del siglo XVII el discurso predominante sobre los indígenas del sur de Chile. Este se revela en el ritual político del requerimiento, donde los indígenas debían reconocer el poder soberano y transformarse en vasallos del rey (ver Todorov, 1985). En este entonces se establecieron los dispositivos de la encomienda, de la esclavitud y de la guerra (Boccara, 1998a, 1998b).

La deducción de la fuerza de trabajo de los indígenas en favor de la corona fue el principal objetivo de la encomienda. Los indígenas no eran siervos, lo que impidió también procesos de re-feudalización en las colonias, sino súbditos que debían pagar un tributo al rey. En Chile la encomienda fue importante hasta fines del siglo XVI para la minería del oro, mientras la apropiación de rendimientos agrícolas fue de menor importancia. Por eso Boccara expone la hipótesis de que en el siglo XVII, cuando los yacimientos de oro se acabaron, fueron sustituidos por la legalización de la esclavitud (ver Boccara, 1998a: 214-217).

Según el esquema jurídico binario, los indígenas tenían que reconocer el poder soberano transformándose en súbditos. Si se negaban se contraponían de forma diametral al poder soberano, haciéndose víctimas de la "guerra justa" y de la "esclavitud legítima". En ambos casos se da el derecho soberano de muerte, siendo la respuesta del soberano a la violación de su querer.

Hay que poner de relieve que la Corona prohibió la esclavitud de los indígenas ya en 1542. En Chile, sin embargo, fue legalizada entre 1608 y 1674. La re-introducción de la esclavitud aseguró primero, la fuerza de trabajo en la minería y en la agricultura y segundo, fue un instrumento estratégico en la lucha contra los mapuches, que resistieron con mucho éxito.

Un segundo dispositivo para la sumisión de los mapuches fue la guerra. Ya en los tiempos primeros de la Conquista, la Corona fue forzada a tener un ejército permanente (Villalobos, 1993: 144), cuya estrategia principal eran los asaltos. llamados malocas. El objetivo de dichos malocas no era la colonización sino la destrucción de las chacras y rucas mapuches y el robo de esclavos. Estos ataques pueden ser interpretados como ritual político para el reestablecimiento del honor violado del soberano que hace sufrir por este motivo a los cuerpos de los indígenas.

5. Eso no quiere decir que son explicables todos los procesos en el espacio politico por la acción del soberano. Sabemos que las negociaciones de poder (p.e., en las cortes) fueron más sutiles y complicadas - pero el poder soberano fue la lógica politica dominante. 
En Chile, la sumisión fracasó sobre todo a causa de la lógica bélica de las sociedades indígenas. 6 La sociedad reche-mapuche parecía una red acéfala de grupos parentales y aliados con un patrón de asentamiento disperso. El modo de conflicto puede ser entendido en términos de un "differend" (Lyotard, 1989) - un conflicto sin escala común. Los españoles pudieron percibir a los indígenas sólo en términos negativos. mientras la sociedad reche-mapuche era determinada por una lógica híbrida de la integración e incorporación de lo ajeno. Siguiendo la tríada guerrero-canibalista de "captar-digéreer-différencier" (Boccara, 1998a) eran asumidos e incorporados elementos ajenos para nutrir y transformar lo propio. Esta lógica híbrida no se limitaba a la guerra sino que también tomaba otros elementos ajenos como el caballo, el trigo, las monedas de plata, etc.. los que eran integrados $y$ transformaban su sociedad.

\section{Resultados}

\section{Poder pastoral y razón del Estado}

Frente al equilibrio militar se heló la Conquista (1650-1850), y el poder pastoral, como razón del Estado, sustituyó al poder soberano. El concepto del poder pastoral fue desarrollado por Foucault en el contexto del proyecto de la gubernamentalidad. Originado en el cristianismo, Foucault lo considera fundamental para la construcción del estado occidental. Especialmente en el siglo XV y XVI, en los tiempos de la reformación y contra-reformación, parte Foucault de una intensificación del poder pastoral en Europa. La idea central del concepto es que el pastor es responsable del bienestar de un rebaño de individuos dispersos, es decir, es responsable de "omnes et singulantim" del grupo y de cada individuo, dando luz a poderes de estructuración estatal y de procesos de sujeción (Foucault, 1994c: 65-93).

En Chile, el inicio del poder pastoral se anunció con el jesuita Luis de Valdivia a principios del siglo XVII quien se opuso a la guerra contra los indígenas y la esclavitud, promoviendo una "guerra defensiva" donde los misionarios serían los agentes principales, no los soldados. En lugar de la sumisión militar-jurídica, eran los seres humanos el objetivo del poder. Tanto individuos como grupos, fueron civilizados, evangelizados y asentados en pueblos; la conquista territorial fue reemplazada por la evangelización de las almas.

Mientras el discurso binario del poder soberano diferenció entre dos polos absolutos, se movió el discurso del poder pastoral en una escala métrica entre los polos extremos -creyente/ civilizado y sin fe/salvaje. Por eso la meta principal de los misionarios era evangelizar y civilizar a los indígenas, luchando de esta manera contra el diablo en favor de sus almas. Para lograr esto tenían que conocer a los mapuches y así crearon la base de un complejo de saber-poder en torno a lo indígena.

Por varias razones, la estrategia de Valdivia fracasó entre 1612 y 1626 . Sin embargo, su racionalidad política fue revitalizada 20 años más tarde por el gobernador Marqués de Baides, cambiándole el sentido (Ver Boccara, 1998a). Mientras la meta de Valdivia era "gobernar las almas", con la Misión, de Baides secularizó el concepto señalando que la razón del Estado era "gobernar los hombres". Frente al equilibrio militar, el punto clave de Baides era la física, la economía de las fuerzas, poniendo la economía del Estado en el centro de las técnicas de gobernar, disponiendo a la vez, del poder soberano.

La transición del poder soberano pasando por el poder pastoral hasta la técnicas de gobernar de la razón del Estado fueron bastante productivas, produciendo un nuevo saber y nuevos dispositivos que se conservaron hasta la independencia chilena. El "arquetipo" (Boccara, 1998a: 227) de las nuevas técnicas de poder era la Misión, que tomó un papel central en las técnicas de dominación desde la mitad del siglo XVII. La Misión instaló una vigilancia permanente sobre los reche-mapuches tanto grupal como individual, investigando los pensamientos y prácticas de cada uno. Técnicas de vigilancia eran la regulación estricta del tiempo (trabajo, misa, etc.) y de la fuerza de trabajo para poder

6. Según Wallerstein, la constitución de un imperio mundial fracasó por motivos económicos y administrativos dando lugar al surgimiento del sistema capitalista mundial en el longue durée del siglo XVI (Wallerstein 1986: 258 pp.) 
aumentar la producción agrícola. Pero muchas veces los mapuches se negaron a la vigilancia, razón por la cual los misioneros trataron de asentar la población en pueblos y misiones. Otra meta de sus técnicas fue la civilización de los hijos de los caciques para evangelizar y civilizarlos terminando con "costumbres paganas" como la vida bélica, la promiscuidad y la gula. Junto con estas técnicas de sujeción se aplicaron también técnicas para el control de la población como estadísticas de migración y cifras de mortalidad y natalidad. El espacio "pagano" tenía que ser controlado por una red de capillas y misiones, significando a la vez una sacralización simbólica del espacio.

A mediados del siglo XVII los parlamentos lugares para negociar las relaciones diplomáticas, políticas y económicas entre mapuches y españoles - llegaron a ser un dispositivo importante. El Parlamento de Quilín que tuvo lugar el 6 de enero de 1641 en las cercanías de Temuco, fue sin duda el "prototipo" (Villalobos, 1992: 387) de este dispositivo que determinó las relaciones inter-étnicas desde la mitad del siglo XVII hasta fines del siglo XIX. En dicho Parlamento, el río Bío-Bío era reconocido como frontera, hecho que inició una etapa que en la visión de los estudios fronterizos fue caracterizada por el florecimiento del negocio y una convivencia pacífica. Sin embargo, con Boccara hay que destacar: "El querer someter a los indigenas nunca desapareció. Se transformó." (Boccara, 1998a: 226). Y esta transformación se debe en gran parte a la lógica de las relaciones de fuerza.

El Parlamento funcionó como dispositivo de un poder pastoral que sujetaba al individuo y a la civilización al control de lo colectivo. Mientras en Europa occidental se formaron las primeras casas de trabajo controlando las masas, se desarrolló un ethos de trabajo moderno y disciplinado y surgió el concepto de delincuente. Los parlamentos en la Araucanía buscaron técnicas semejantes para el control de población, articulando el fenómeno del vagabundismo en el valle central y los mapuches no-controlables de la Araucanía. De ambos elementos surgió la imagen del delincuente en
Chile. Asi como la Misión produjo al "salvaje". el Parlamento produjo al delincuente que debía ser procesado por la ley occidental.

En los acuerdos del Parlamento de Tapihue (1774), fueron combinados técnicas de vigilancia con un discurso juńdico que hacía responsable individualmente a los caciques por cualquier acto de delincuencia en sus territorios. Se trata de una regulación específica y una bio-política en cuyo margen el cacique llegó a ser el agente del auto-control mapuche.

A pesar de esta racionalidad política, no debemos olvidar que los mapuches no fueron sometidos por los españoles y que las técnicas y estrategias del poder pastoral y de la razón del Estado no se aplicaron de una forma pura. Además hay que recalcar que la sociedad reche-mapuche pudo mantener su autonomía relativa viviendo cambios profundos. En complejos procesos de constitución propia y ajena nació la nueva etnia de los mapuches (Boccara, 1998a, 1999a, 1999b). Bajo las condiciones de una "conquista congelada" lograron ampliar su territorio apropiándose de la pampa argentina, llegando a ser ganaderos, viviendo del ganado cimarrón de la pampa y de la producción de textiles. Los mapuches tejieron redes de intercambio y comercio con los españoles de la región fronteriza, siendo sus productos más importantes, ganado, textiles y sal.

Dentro de la sociedad mapuche se dio la tendencia de centralizar el poder político en las manos de los ülmen. Este proceso fue acompañado por una nueva definición de imágenes geopolíticas, institucionalizando unidades político-espaciales como los ayllarehue (nueve rehue) y las futamapu.

Esta formación de poder está caracterizada por la ausencia de una guerra abierta, formas de poder y dominación más sutiles y transformaciones enormes dentro de la sociedad mapuche. Geopolíticamente se constituyó un régimen fronterizo estable teniendo el Bío-Bío como frontera reconocida también por la Corona Española. Hasta la Conquista de parte 
del Estado chileno, podemos hablar de dos sociedades autónomas que se influenciaron mutualmente.

\section{El sueño de la "comunidad pura"}

Además de razones político-económicas de colonización de la Araucanía no-capitalista para la producción de trigo de exportación, estaba el sueño político de la "comunidad pura" (Foucault 1998a: 202), que a fines del siglo XIX formó la base de la conquista militar de la Araucanía. A mediados del siglo XIX el EstadoNacion chileno se consolidó después de la Independencia en 1818. Ahora la gran meta era terminar el proyecto moderno del orden, separando lo no-controlable y lo caótico, parcelando el territorio de la Araucanía, integrándolo al proceso de la acumulación capitalista. En 1866 empezó la segunda conquista militar. De esta manera, la formación del poder soberano trató de exterminar al mapuche, no sólo de someterlo. Se formó un discurso racista que estableció primero una jerarquía de las razas humanas, surgiendo luego la relación siguiente: "La muerte del otro - en la medida en que representa mi seguridad personal - no coincide simplemente con mi vida. La muerte del otro, la muerte de la mala raza, de la raza inferior (o del degenerado o del inferior) es lo que hará la vida más sana y más pura." (Foucault 1994b: 18) En el caso de los mapuches en Chile significó esto: La "raza inferior" de los Mapuche debe ser eliminada para no obstaculizar el desarrollo de la "raza superior" de los chilenos. Esta argumentación está cimentada por un modelo histórico-filosófico de desarrollo en cuyo margen los chilenos se vieron en la vanguardia de un desarrollo universal. En este sentido, el intelectual chileno Benjamín Vicuña Mackenna argumentó, en el debate parlamentario sobre la política de la conquista de la Araucanía, que la historia había demostrado que el indio "no era sino un bruto indomable, enemigo de la civilización, porque sólo adora los vicios en que vive sumergido, la ociosidad, la embriaguez, la mentira, la traición y todo ese conjunto de abominaciones que constituyen la vida salvaje." (Vicuña Mackenna, citado en Pinto, 1998: 101). En este discurso podemos observar una inversión: en vez de ser víctimas, los mapuches llegan a ser culpables, obstaculizando el desarrollo y progreso de los chilenos $y$, por fin, de toda la humanidad civilizada.

Este racismo está vinculado estrechamente al Estado, que llegó a ser el agente principal de la bio-política, apuntando a la exterminación genocida de los mapuches. Después de la derrota de 1883 ganó importancia el modo de exclusión en el contexto del "sueño de la comunidad pura" (Foucault, 1998a). El "Estado jardinero" (Barman, 1995. 43-65) que ordena su territorio nacional limpiándolo de maleza, transplanta los mapuches a territorios marginales de la cordillera de la costa y andina para tenerlos a distancia de la comunidad pura. Las unidades socio-políticas de los mapuches fueron desarticuladas y ellos fueron segregados a reducciones - lugares aparte de la sociedad chilena. El espacio conquistado debería ser "replantado"con colonos europeos para "mejorar la sangre" del "cuerpo de la sociedad chilena". Era la intención de impedir el desarrollo de un latifundismo "feudal" fomentando campesinos medianos según el ejemplo europeo. A pesar de esto no tardó mucho para que la hacienda entrara a La Araucanía.

La derrota militar significó el fin de la sociedad mapuche. La sociedad autónoma que tenía relaciones comerciales con los chilenos y que amplió su territorio hasta la pampa, fue destrozada en más que 3.000 reducciones, transformando a los ganaderos en agricultores de subsistencia.

A principios del siglo XX podemos destacar dos movimientos de resistencia de los mapuches frente a esta situación. Por un lado, los mapuches se apropiaron de las reducciones, que inicialmente fueron creadas para su exclusión, creando verdaderas comunidades de resistencia (Bengoa, 1996). Estas comunidades no son restos tradicionales de una cultura antigua mapuche, sino por el contrario respuestas actuales a la modernización y al dominio capitalista. (Ver Kaltmeier, 2004).

7. Foucaulf (1998a) describe la genealogía del "sueño de la comunidad pura" con el ejemplo de los rituales de exclusión, los cuales fueron en el siglo XVII la respuesta a la lepra. 
Por otro lado, menos de 30 años después de la derrota militar se formaron organizaciones mapuches que pueden ser entendidas en términos de movimiento social. Al contrario de las comunidades (auto-)excluidas, este movimiento se dirigió a la sociedad chilena. Se entendió como elemento integral de la sociedad chilena para influenciar autorreflexivamente a dicha sociedad. Hasta mitades de los años 50 era determinante la Corporación Araucana que combatió el racismo y que fomentó la integración de los mapuches a la sociedad chilena, moviéndose en el margen del indigenismo. Era una lucha por el reconocimiento de la sociedad presente.

\section{La "sociedad disciplinada"}

A finales de los años 20 el sueño de la comunidad pura terminó y las estrategias de exterminación y exclusión fueron reemplazadas por una "política más específica y auténtica del EstadoNación" (Bauman 1995: 94): la asimilación. La "sociedad disciplinada" (Foucault 1998a: 202) llegó a ser el nuevo ideal del imaginario nacional. Por lo tanto las reducciones en el corazón del territorio chileno debían ser destruidas, aplicando las técnicas de una disciplina parceladora al espacio de exclusión de las reducciones con el motivo de integrar a los mapuches en función de campesinos pobres con parcelas privadas, bien medidas. Entre 1927 y 1970 surgieron varias leyes con el fin de dividir las comunidades, disolver la propiedad colectiva, establecer la propiedad privada y desarticular a las comunidades de resistencia.

El intento de la parcelización de la comunidad fue acompañado por politicas disciplinadoras y normalizantes en los sectores de salud, educación, agropecuario e instituciones como el servicio militar. A pesar de esto más que dos tercios de las comunidades pudieron resistir hasta 1971 al proceso de división. (González 1986: 7-13)

La lógica de gobernar bajo la Unidad Popular de 1970 a 1973 también fue caracterizada por una política asimiladora moderna en el sentido del indigenismo, asimilando lo indígena a la nación mestiza. Sin embargo el sentido político era diferente. La política de la Unidad Popular (UP) no se concentró en la conservación del estatus quo y la satisfacción de los intereses de las elites, sino en la transición al socialismo en cuyo margen los subalternos ganaron más derechos y recursos. Basta mencionar la destrucción del dispositivo de la hacienda por medio de la realización consecuente de la reforma agraria (tierra para mapuches, campesinos pequeños e inquilinos), la introducción de una ley indígena que por primera vez en la historia chilena reconoce a los mapuches asegurando su propiedad colectiva y aplicando diversos programas sociales y educativos.

En el contexto de un cambio latinoamericano hacia demandas revolucionarias, se formó en los años 30 y, más fuerte en los años 50 , una lucha redistribuidora-campesina a la que se unieron partes del movimiento mapuche. El objetivo era la integración a la sociedad chilena. pero en diferencia al discurso de reconocimiento de los primeros años, este estaba vinculado a la demanda de cambios socio-económicos sobre todo una reforma agraria; temas en torno al reconocimiento o a diferencias culturales quedaron atrás. Bajo la UP se vinculó la política estatal-aunque sería exagerado hablar de una explícita política indígena de la UP - con la lucha redistribuidora del movimiento mapuche, dejando a un lado los temas de reconocimiento cultural.

\section{Dictadura y gobierno neoliberal}

El bombardeo del Palacio presidencial, La Moneda, no fue sólo la destrucción de un lugar material sino una señal simbólica. Los militares golpistas alrededor de Augusto Pinochet demostraron que no sólo terminarian con el gobierno de la Unidad Popular sino con todas las tradiciones democrático- populistas anteriores. En este sentido, la Junta, después de pequeñas discusiones internas, no optó por reestablecer el orden antiguo, sino que realizó una verdadera "revolución capitalista" 
introduciendo un modelo neoliberal que antes había existido solo en los libros de la escuela económica de Chicago.

El sociólogo Tomás Moulian divide la época de la dictadura en dos fases (Moulian, 1997). En la primera fase, entre 1973 y 1980, dominó el dispositivo del terror aniquilando casi todo tipo de resistencia e introduciendo una subjetivación atomista. En el dispositivo del terror se entrelazan la visibilidad del castigo con la invisibilidad de la disciplina. El terror necesita una visibilidad permanente para poder inscribirse en las almas de los hombres, aplicando en el caso chileno especialmente la "invisibilidad visible" de los detenidos-desaparecidos, vigente hasta ahora. La arbitrariedad y la visibilidad permanente del terror hacian de toda acción un riesgo, especialmente en el espacio público. Empezó un recogimiento hacia lo privado, una migración interna, que debilitó formas solidarias. Estas nuevas subjetivaciones creadas por el terror fueron una base para la imagen neoliberal del homo económico.

En la segunda fase a partir de 1980 hasta el fin de la dictadura en 1990, se institucionalizó la dictadura. Basta mencionar la nueva Constitución de 1980, pero también algunas leyes anteriores, como la ley indígena o la ley laboral que construyeron la base de la nueva gubernamentalidad neoliberal. Sería falso reducir las nuevas formas de gobernar a la fórmula "más mercado - menos Estado" porque no se trata de una relación cuantitativa sino de otro programa de gobernar, de una racionalidad política cualitativamente diferente. Foucault argumenta que lo económico coloniza lo social, desapareciendo por fin lo social. (Ver Foucault, 2004b: 340) Lo económico llega a ser la única racionalidad válida y al fin está naturalizada. En este aspecto le damos la razón a Valdés argumentando que el neoliberalismo llegó a ser la "filosofía fundadora de una nueva sociedad" (Valdés, 1993: 57).

La gubernamentalidad neoliberal implica formas especificas de sujetización que se expresan en el homo económico, entendido como destino natural del hombre. Para el mapuche significaría esto transformarse en campesino, maximizando sus beneficios, el que para poder trabajar en "libertad", debería ser liberado de sus obstáculos, como la propiedad colectiva que contradice a la naturaleza del hombre. Así, la propiedad colectiva fue abolida, la comunidad dividida y eliminada la categoría socio-cultural del indígena. Dice la ley: "las hijuelas resultantes de la división de las reservas dejarán de considerarse tierras indígenas e indigenas sus dueños y adjudicatarios" (Decreto ley No. 2568, citado en Aylwin, 1990: 346). Entonces el objetivo de la ley no sólo fue la transformación de la propiedad colectiva en propiedad privada, sino también normalizar /chilenizar a los indígenas y constituirlos como sujetos del mercado.

Tal como para otros movimientos sociales, el "Golpe" fue una verdadera censura para el movimiento mapuche: fueron desarticuladas sus organizaciones, detenidos, exiliados y/o eliminados sus dirigentes; las recuperaciones de tierras deshechas y la política de reconocimiento limitada sustituida por una ley indígena etnicitaria. Sólo a fines de los años 70 , se iniciaron, con los Centros Culturales Mapuches y después con Ad-Mapu, los primeros intentos reorganizativos en contra de la ley indígena de la dictadura. Sin embargo, estas nuevas organizaciones no se relacionaron con la lucha redistribuidora-campesina sino que acentuaron el derecho a la diferencia cultural. Primeramente este movimiento tenía estrechos vínculos con las comunidades, pero en el transcurso de la Dictadura el movimiento advirtió que no sería posible realizar la lucha por el reconocimiento cultural. Por esto, y por el empobrecimiento creciente, el movimiento mapuche se articuló con otros movimientos antidictatoriales, llevando una lucha popular (Laclau y Mouffe, 2000: 172). Con eso el campo de la lucha cambió de las comunidades hacia la sociedad nacional, abriéndose otra vez la brecha entre comunidades y el movimiento mapuche. 


\section{Transición y transformismo}

Los gobiernos post-dictatoriales no abandonaron el modelo neoliberal sino que, especialmente los gobiernos de Eduardo Frei Ruiz-Tagle (1994 2000) y de Ricardo Lagos (desde 2000), iniciaron una tercera fase de la implementación del modelo. El primer pobierno post-dictatorial de Patricio Aylwin (1990-1994) firmó un "doble contrato social": Por un lado cedió a ciertas demandas de los movimientos populares, conduciendo, en el caso mapuche a través del Acuerdo de Nueva Imperial, a una nueva Ley Indígena y a la Corporación Nacional de Desarrollo Indígena (CONADI). Por otro lado se mantuvo la tríada dominante (Moulian, 1997: 18) de militares, empresarios e intelectuales neoliberales en el poder, pues, la Concertación se vio obligada a continuar el modelo económico introducido por la dictadura, mantener la constitución de 1980 y asegurar la impunidad para los militares, sus ayudantes y sobre todo para Pinochet.

Bajo esta luz, la transición parece un transformismo, 9 la continuación del modelo de la dictadura bajo condiciones democráticas. Lo nuevo de los gobiernos post-dictatoriales es la introducción y amplificación de la racionalidad política neoliberal y su institucionalización. Mientras la dictadura erradicó lo social y se concentró sobre todo en aspectos económicos y estructurales, es tema central de los gobiernos post-dictatoriales, el campo político-cultural.

El Chile actual se entiende como liberado de los fantasmas de la dictadura. Es una sociedad sin historia, basada en el juego del mercado aspirando a un desarrollo económico futuro. El discurso hegemonial consiste en una cadena de equivalentes: proyecto neoliberal = apertura económica $=$ crecimiento económico $=$ empleo $=$ menos pobreza $=$ crecimiento con equidad $=$ desarrollo regional = segunda etapa de exportación = consenso nacional = amplificación de la democracia (Agacino, 1999: 71-84).

El reformismo tenía coyuntura y un modelo liberal de negociar conflictos sociales fue establecido en todos los campos políticos expresándose en nuevas instituciones como Servicio Nacional de la Mujer (SERNAM), Corporación Nacional del Medioambiente (CONAMA) o CONADI. Estas instituciones comparten cierto carácter híbrido, porque su origen se debe, por un lado, a la iniciativa de los movimientos sociales mientras, por otro lado, contribuyen a la gubernamentalización neoliberal del Estado, congelando conflictos sociales, canalizándolos a vías institucionales, administrándolos y negociándolos.10

En este contexto, también se dislocó la contradicción entre movimientos sociales y Estado. En vez de constatar un antagonismo argumentamos que ambos, movimientos sociales y Estado, son partes integrales en la transformación de las técnicas de gobernar; en tal medida que sectores importantes de los movimientos, llegaron a ser parte del Estado.

La lucha cultural de reconocimiento de los mapuche se redujo en dos sentidos: Por un lado, los mapuche no tenían un proyecto alternativo así que casi sólo se dirigían al Estado que quedó en la posición soberana de otorgar reconocimiento o negarlo. Las demandas mapuches llevaron a un "mainstream multiculturalismo" (Fraser 2001: 47), que reconoció a los mapuche como grupo pero que no atacó las relaciones de poder. Por otro lado, la desigualdad socio-económica fue excluida, descuidando el emprobrecimiento de las comunidades mapuche.

En vez de disciplinar el cuerpo o sujetarlo bajo la norma el discurso neoliberal, apunta a la conducción de individuos, para que participen en el juego libre de las fuerzas del mercado. En este aspecto no se trata de la "cultivación y optimización de diferencias" (Lemke, 1997: 251)

Este es el caso de los mapuche que deben constituirse como sujetos del mercado bajo el lema "desarrollo con identidad" y a través de programas de etno-desarrollo. Llama la atención que la nueva ley indígena pone mucho énfasis en otorgarle la personalidad jurídica a las comunidades o nuevas asociaciones indígenas posibilitándoles ejecutar proyectos de desarrollo.

9. Al contrario de la hegemonía expansiva el transformismo es - en la obra de Gramsci- una estrategia defensiva de la burguesía en tiempos de una crisis política económica para la cooptación de grupos antagonistas y movimientos sociales con el fin de crear un consenso pasivo. (Gramsci 1991: 78-79).

10. Este aspecto lo muestra muy bien Verónica Schild en el movimiento feminista en Chile (Ver Schild, 2004, 1998). 
En este contexto, también debe ser observada la última moda a través del uso del concepto del capital social fomentado por el Banco Mundial, especialmente en vista de las comunidades indígenas.

La idea es que en las comunidades se ubiquen importantes reservas de capital social. Para poder aprovechar este capital social los comuneros deben ser empoderados y guiados por expertos. Esto lleva a la construcción de nuevos mecanismos de control y de nuevas sujeciones neoliberales de auto-activación que se basan en el ethos, la moral, y la auto-estima de la comunidad. Depende en última instancia de la misma comunidad si hay desarrollo o no. La comunidad llegó a ser un punto privilegiado de referencia en las prácticas discursivas y nodiscursivas como programas de intervención social o de desarrollo.

A fines de los 90 era obvio que la política del reconocimiento parcial había fracasado. también en vista del agotamiento del modelo neoliberal después de los años del boom en esa década. El reconocimiento cultural quedó limitado (sin suscripción del convenio 169 de la OIT y del reconocimiento constitucional como pueblol y la dimensión de justicia socioeconómica se agravó con la falta de tierra, conflictos con las forestales y proyectos de infraestructura, cesantía, la crisis de la agricultura campesina. Contra estas dimensiones de injusticia se levantó a fines de los noventa un nuevo movimiento mapuche con una lucha por la diferencia, buscando tanto el reconocimiento cultural como redistribuciones socio-económicas. Importante es su nueva política identitaria y territorial, sus formas de lucha que rompen con el sistema de negociaciones, tratando de entrar, a través de la confrontación, a un diálogo.

\section{Conclusiones}

Con este ensayo recorrimos la historia del pueblo mapuche desde la conquista española pasando por la nueva conquista chilena hasta la situación actual. Sin lugar a dudas, hemos simplificado mucho, ya que cada época tiene sus características y ciertas diferencias locales poniendo de esta manera en duda nuestra visión más global. Es un peligro serio generar categorías generales negando las específicas. Por eso queremos destacar que no hemos llevado a cabo una explicación completa y cerrada, sino ofrecido un marco de entendimiento a través del uso de conceptos orientados en los estudios de poder y de la gubernamentalidad de Michel Foucault. Insistimos en la importancia de hacer estudios más locales, porque estamos convencidos de que "el poder" no funciona igual en todos los contextos locales, sino que es flexible y creativo, dando lugar a rasgos diferentes.

Esto nos lleva a un segundo comentario, que acentúa la relación entre racionalidad política y realidad social. El mayor interés de Foucault es el nivel de la racionalidad política, de lo pensable, del saber y su relación con el poder. Siguiendo esta línea, muchos trabajos de los governmentality-studies anglo-americanos se concentran en este nivel y-a diferencia de Foucault-proponen una traducción directa de los programas a la realidad social y a procesos de sujeción. Esto lleva por fin a un modelo teórico que otorga a los programas / la racionalidad política, el estatus de un meta-sujeto, un metaactor, determinando todas las prácticas discursivas y no-discursivas. Para nosotros, en cambio, es la gubernamentalidad la estructuración del campo de acción posible, lo cual es similar a la noción del campo (político) del sociólogo Pierre Bourdieu como arena de luchas (simbólicas).

Esto significa también tomar en serio el dicho de Foucault "dónde hay poder hay resistencia". En el trabajo de Foucault vemos especialmente en la noción de las formas de contra-conducta que él describe como fugas, descentralizaciones, reacciones al poder pastoral en su lectura del 1 de marzo de 1978. En este trabajo no pudimos referirnos sistemáticamente a las formas de contra-conductas de los mapuches, pero terminamos cada capítulo con comentarios sobre ellas.

11. Se puede detectar al menos dos posiciones que predominan en los análisis sobre capital social. La primera es la de Pierre Bourdieu que analiza el capital social y el sistema de relaciones sociales. La segunda define el capital social como una estrategia individual para maximizar los intereses. Destaca que capital social es un factor importante para desarrollo económico. Esta posición está vinculada con los nombres de Coleman y Putnam. En el Banco Mundial está utilizada la segunda postura teórica. 
Esto nos lleva a la pregunta sobre la importancia de la racionalidad política del Otro, la influencia de la diferencia cultural. èHasta qué punto puede influenciar "lo indígena" las modalidades de la gubernamentalidad? (Ver Slater, 1998: 389; O'Malley, 1996) Esta pregunta es de relevancia histórica y también de importancia para el entendimiento de los procesos actuales, pues a partir de los noventa somos testigos de una intensificación inmensa de los programas, instituciones, proyectos etc. que enfocan directamente a los pueblos indígenas como beneficiarios que suponemos se orientan por el fin estratégico de incluirlos a una gubernamentabilidad neoliberal emergente.

\section{Bibliografía}

AGACINO, R. (1999), "Chile 25 Jahre danachVon Licht und Schatten einer reifen Gegenrevolution", en KALTMEIER, O. y RAMMINGER, M. (Ed.), Links von Nord und Süd, Münster: 66-86.

AYLWIN, J. (1990), "Tierra Mapuche-derecho consuetudinario y legislación chilena", en STAVENHAGEN, R. y ITURRALDE, D. (Ed.), Entre la ley y la costumbre, México: 333-354.

BAUMAN, Z. (1995), Moderne und Ambivalenz, Frankfurt a.M..

BENGOA, J. (1996), Historia del Pueblo Mapuche, Santiago.

BOCCARA, G. (1998a), Guerre et ethnogènese mapuche dans le Chili colonial, Paris.

BOCCARA, G. (1998b), "Dispositivos de poder en la sociedad colonial-fronteriza chilena del siglo XVI al siglo XVIII", en PINTO, J. (Ed.), Del discurso colonial al proindigenismo. Ensayos de Historia Latinoamericana, Temuco: 29-42.

BOCCARA, G. (1999a), "Organisation sociale, guerre de captation et ethnogenèse chez les Reche-Mapuche à l'époque coloniale", en L'Homme 150: 85-117.
BOCCARA, G. (1999b), "Etnogénesis mapuche: resistencia y restructuración entre los indígenas del centro-sur de chile (siglos XVI-XVIII)", en Hispanic American Historical Review 3: 425-461.

BOCCARA, G. (2000), "Antropología diacrónica. Dinámicas culturales, procesos históricos y poder político", en BOCCARA, G. Y GALINDO, S. (Ed.): Lógica mestiza en América. Temuco: 21-59.

FOUCAULT, M. (2004a), Geschichte der Gouvernementalität I. Sicherheit, Territorium, Bevölkerung, Frankfurt a.M.

FOUCAULT, M. (2004b), Geschichte der Gouvernementalität II. Die Geburt der Biopolitik, Frankfurt a.M.

FOUCAULT, M. (1999), In Verteidigung der Gesellschaft, Frankfurt a.M..

FOUCAULT, M. (1994a) "Omnes et singulantim. Zu einer Kritik der politischen Vernunft", en VOGL, J. (Ed.), Gemeinschaften. Positionen zu einer Philosophie des Politischen, Frankfurt a.M.: 6593.

FOUCAULT, M. (1994b) "Leben machen und sterben lassen. Die Geburt des Rassismus", en Tüte, suplemento Wissen und Macht, Tübingen: 15-19.

FOUCAULT, M. (1998a), Vigilar y Castigar, México.

FOUCAULT, M. (1998b), La voluntad de saber, México.

FOUCAULT, M. (1985), Freiheit und Selbstsorge, Frankfurt a.M..

FRASER, N. (2001), Die halbierte Gerechtigkeit. Gender Studies, Frankfurt a.M..

GONZÁLEZ, H. (1986), "Propiedad comunitaria o individual - Las leyes indígenas y el pueblo mapuche", en Nütram 3: 7-13. 
GRAMSCI, A (1991) "Das Problem der politischen Führung bei der Bildung und Entwicklung der Nation und des modernen Staates in Italien" en NEUBERT, H. (Ed.), Antonio Gramsci-vergessener Humanist? Berlin: 77-102

KALTMEIER, O. (2004), "iMarichiweu! Zehnmal werden wir siegen! Eine Rekonstruktion der aktuellen Mapuche-Bewegung in Chile aus der Dialektik von Herrschaft und Widerstand seit der Conquista", Münster.

LACLAU, E. y MOUFFE, CH. (2000), Hegemonie und radikale Demokratie. Zur Dekonstruktion des Marxismus, Wien.

LEMKE, TH. (1997), Eine Kritik der politischen Vernunft. Foucaults Analyse der modernen Gouvernementalität, Hamburg.

LEMKE, TH. (2002), ""Die verrückteste Form der Revolte" - Michei Foucault und die Iranische Revolution", en 1999. Zeitschrift für Sozialgeschichte des 20 . und 21 . Jahrhunderts 2: 73-99.

LYOTARD, F. (1989), Der Widerstreit. München.

MOULIAN, T. (1997), Chile Actual. Anatomía de un mito, Santiago de Chile.

MUÑOZ PÉREZ, J. (1986) "Regierung und Verwaltung von Spanisch-Amerika", en, Gold und Macht. Spanien in der Neven Welt. (Catálogo de la exposición), Wien: 116-124.

O'MALLEY, P. (1996) "Indigenous governance", en Economy and Society 3: 310-326.

PINTO, J. (1998), "Del antiindigenismo al proindigenismo en Chile en el siglo XIX", en PINTO, J. (Ed.), Del discurso colonial al proindigenismo. Ensayos de Historia Latinoamericana. Temuco

SCHILD, V. (1998) "New Subjects of Rights? Women's Movements and the Construction of Citizenship in the "New Democraciesu.", en ALVAREZ, S., DAGNINO, E. y ESCOBAR, A. (Ed.). Cultures of Politics - Politics of Cultures. Revisioning Latin American Social Movements. Boulder: 93-117.
SCHILD, V. (2004) "Die Freiheit der Frauen und gesellschaftlicher Fortschritt. Feministinnen, der Staat und die Armen bei der Schaffung neoliberaler Gouvernementalität", en KALTMEIER, O., KASTNER, J. y TUIDER, E. (Ed.), Neoliberalismus - Autonomie - Widerstand. Analysen Sozialer Bewegungen in Lateinamerika. Münster.

SLATER, D. (1998), "Rethinking the Spatialities of Social Movements: Questions of (B)orders, Culture, and Politics in Global Times", en ALVAREZ. S., DAGNINO, E. y ESCOBAR, A. (Ed.), Cultures of Politics - Politics of Cultures. Re-visioning Latin American Social Movements, Boulder: 380-401.

TODOROV, T (1985), Die Eroberung Amerikas. Das Problem des Anderen, Frankfurt a.M..

VALDÉS, J. (1993) "Die Chicago-Schule: Operation Chile", en Lateinamerika . Analysen und Berichte 17: 36-60.

VILLALOBOS, S. (1992), La vida fronteriza en Chile, Madrid.

WALLERSTEIN, I. (1986), Das moderne Weltsystem. Kapitalistische Landwirtschaft und die Entstehung der europäischen Weltwirtschaft im 16. Jahrhundert, Frankfurt a.M.. 